\title{
Optimal Control Strategy for the Transmission Dynamics of Typhoid Fever
}

\author{
Temesgen Debas Awoke \\ Department of Mathematics, College of Natural and Computational Science, Kotebe Metropolitan University, Addis Ababa, Ethiopia \\ Email address: \\ temesgendebas@mtu.edu.et \\ To cite this article: \\ Temesgen Debas Awoke. Optimal Control Strategy for the Transmission Dynamics of Typhoid Fever. American Journal of Applied \\ Mathematics. Vol. 7, No. 2, 2019, pp. 37-48. doi: 10.11648/j.ajam.20190702.11
}

Received: April 13, 2019; Accepted: May 28, 2019; Published: June 26, 2019

\begin{abstract}
The author developed a deterministic mathematical model for Typhoid fever disease dynamics that accounts for Vaccination and relapse of treatment. Three control strategies (vaccination, treatment of infection, screening and treatment of carriers) are applied to investigate the optimal intervention strategy of controlling Typhoid disease transmission. The aim of this study is to determine the optimal combination strategy of vaccination, treatment of infection, screening and treatment of carriers that will minimize the cost of those strategies and the number of Infective and Carriers. The author used Pontryagin's maximum principle to characterize the optimal level of those three strategies. The result is simulated numerically using RungeKutta fourth order method through MATLAB software. Numerical results showed that implementation of all controls or a combination of vaccination, treatment of invectives as well as screening and treatment of carriers is the best strategy to eradicate the disease at an optimal level with minimum cost of interventions.
\end{abstract}

Keywords: Typhoid Fever, Optimal Control, Pontryagin Maximum Principle, Equilibrium Point, Basic Reproduction Number, Numerical Simulation

\section{Introduction}

Typhoid fever is an endemic infectious disease caused by a highly virulent and invasive Salmonella entericaserovarTyphi (S. Typhi) that affects humanity $[1,2]$. The bacteria are transmitted directly from human to human and indirectly from the environment to human through food and water contaminated with faeces and urine of an infected patient or a carrier [3]. Signs and symptoms include; sustained fever, poor appetite, vomiting, severe headache and fatigue.

The transmission of Typhoid fever can be prevented and controlled through Vaccination, safe drinking water, improved sanitation and medical treatment with oral Chloramphenicol, and Amoxicillin [2]. Furthermore, the chronic carrier state may be eradicated using oral therapy using Ciprooxacin or Noroxacin. Multi-drug resistant strain of Salmonella Typhi are increasingly common worldwide, which makes treatment by antibiotics more difficult and require high cost specially in developing countries as indicated from a study[4] that Typhoid fever affects millions of people worldwide each year, where over 20 million cases are reported and kills approximately 200,000 annually. For instance, in Africa it is estimated that 400,000 cases occur annually, an incidence of 50 per 100,000 [3].

Mathematical modeling continues to play a significant role in epidemiology by providing deeper insight into the underlying mechanisms for the spread of emerging and reemerging infectious diseases and suggesting effective control strategies [5-10]. The successful eradication of these emerging diseases does not depend only on the availability of medical infrastructures, but also on the ability to understand the transmission dynamics of a particular disease and the application of optimal control strategies and the implementation of logistic policies [6]. Mathematical models have been used in comparing, planning, implementing, evaluating, and optimizing various detection, prevention, therapy, and control programs. A number of Mathematical models of infectious disease dynamics have been developed and analyzed to determine the impact of Vaccination and treatment [11-13]. In particular, the following authors [14-16] developed a mathematical model for The transmission dynamics of Typhoid fever disease to evaluate the impacts of Vaccination on preventing the disease transmission. Asymptomatic carriers are believed to play an essential role 
in the evolution and global transmission of Typhi, and their presence greatly hinders the eradication of Typhoid fever using treatment and vaccination [1]. The impacts of carriers on the transmission dynamics of Typhoid fever disease have also been also analyzed by these studies $[17,18]$. In addition to this, the roles of Vaccination, treatment of infectious individuals and that of carriers have been studied by different scholars [11, 16, 19-21]. The role of optimal control strategy for controlling the transmission of different disease dynamics have been studied by different authors such as these studies[5, 11, 13]. But the combined optimal control strategies of all the above preventive and control strategies for Typhoid fever, which are prevention with vaccination, treatment of infections, screening and treatment of carriers has not been studied by applying optimal control concepts to determine the best strategy with minimum cost of intervention. Hence, the general objective of this study is to determine or find the optimal combination strategy of vaccination, treatment of infections, screening and treatment of carriers that will minimize the cost of those three control measures and at the same time minimize the number of infective and carriers.

The model formulation and its basic properties are given in the next section. The basic reproduction number, disease free and endemic equilibrium points are derived and discussed in Section 3. Formulations of optimal controls, existence of optimal solutions as well as the derivation of Hamiltonian equation to proof the necessary conditions have been discussed in section 4. In Section 5, numerical simulation of Typhoid disease transmission, the roles of each control measures are discussed. Summery and general recommendations round up the paper.

\section{Model Formulation and Basic Properties}

We formulate a mathematical model that describes the dynamics of typhoid infection in a population. The model subdivides the total population $(\mathrm{N})$ into six sub-population or compartments depending on the epidemiological status of individuals. These are, Susceptible (S), Vaccination (V), Invective (I), carriers $\left(\mathrm{I}_{\mathrm{C}}\right)$, Treated invectives $(\mathrm{T})$ and recovered individuals $(\mathrm{R})$. Thus, the total population is given by $\mathrm{N}=\mathrm{S}+\mathrm{V}+\mathrm{I}+\mathrm{I}_{\mathrm{C}}+\mathrm{T}+\mathrm{R}$. Assume that there is a constant recruitment rate $\pi$. (into the susceptible class) and a per capita natural death rate $\mu$. The dynamics of typhoid transmission will look like as follows.

Susceptible individuals may be vaccinated with a rate $\phi$ and vaccinated individuals join non-vaccinated class at a rate $\omega$. Due to some reasons such as the type of the vaccine used and genetic makeup of individuals, we assume that the vaccine efficacy varies [11]. Then the model assumes that the vaccination is not $100 \%$ efficient and as such, individuals in this class can be infected via contact with individuals in the infected class $\mathrm{I}$, but at a lower rate $\lambda \varepsilon$. Thus $(1-\varepsilon)$ measures the failure of the vaccine in preventing infection with $0 \leq \mathcal{E} \leq 1$. Here, $\mathcal{E}=0$ implies that the vaccine is not effective at all while $\mathcal{E}=1$ means the vaccine renders $100 \%$ protection. Hence, due to failure of the vaccine, vaccinated individual contract the disease with $\lambda$ (force of infection). If $\rho$ proportion of susceptible individuals become new infected symptomatically with the bacteria, then the remaining (1- $\rho$ ) proportion become carriers. The force of infection $(\lambda)$ for contracting the disease is given by $\lambda=\frac{c \beta\left(I+k_{1} I_{c}+k_{2} T\right)}{N}$ and the modified parameters $k_{1}$ and $k_{2}$ accounts for infectiousness of individuals that satisfy $\mathrm{k}_{1} \leq 1 \leq \mathrm{k}_{2}$ and also $\beta$ and $\mathrm{c}$ are the probabilities of transmission and the average number of contacts an individual can make with infectious individuals respectively. This is to show the assumption that the rate of transmission of carriers is high as compared to other infectious individuals. Every compartment decreased due to natural death at a rate $\mu$. In addition to this, infectious and treated classes further decreased due to disease induced deaths at a constant rate $\delta_{1}$ and $\delta_{2}$ respectively. Infectious classes decreased by joining treatment class with constant rate $\eta$. Similarly, carrier individuals join treatment class with a rate $\sigma$. In addition, those infected people will recover from infection with constant rate $\alpha$ and join recovered class $\mathrm{R}$ without receiving treatment. Treated individuals can be decreased by complete recovery with rate $\gamma$ and will join R class or will join a carrier class due to relapse at a rate $\gamma$. Treated individuals recover faster at a rate $\gamma$ as compared to those who do not receive treatment so that $\gamma>\alpha$. We also assume that the carriers develop symptoms at a constant rate $\theta$ and proceed to symptomatic invectives. We assume that the rate of transmission of carriers is high as compared to other infectious individuals as may be unaware of their disease status followed by symptomatic invectives and consequently treated individuals. We also assume that recovered individuals are permanently immune and it assumes that upon treatment, a fraction of individuals relapse and become carriers (primary reservoirs of the disease) and contribute to the transmission dynamics of the disease. With the above assumptions, terminology and interrelations between the parameters and variables, the dynamics of the typhoid fever model can be described by the following deterministic system of nonlinear ODE:

$$
\begin{aligned}
& \dot{V}(t)=\phi S(t)-(\omega+(1-\varepsilon) \lambda+\mu) V(t) \\
& \dot{S}(t)=\pi+\omega V(t)-(\mu+\lambda+\phi) S(t) \\
& \dot{I}(t)=\rho \lambda S(t)+(1-\epsilon) \lambda V(t)+\theta I_{C}(t)-A_{1} I(t) \\
& \dot{I}_{C}(t)=(1-\rho) \lambda S(t)+\tau T(t)-A_{2} I_{C}(t) \\
& \dot{T}(t)=\eta I(t)+\sigma I_{C}(t)-A_{3} T(t) \\
& \dot{R}(t)=\alpha I(t)+\gamma T(t)-\mu R(t)
\end{aligned}
$$

Where,

$$
A_{1}=\delta_{1}+\mu+\alpha+\eta, \quad A_{2}=\theta+\sigma+\mu, A_{3}=\mu+\gamma+\tau+\delta
$$

Boundedness of the Solution

Consider the following biological feasible region. 
$\Omega=\left\{\left(\mathrm{S}, \mathrm{V}, \mathrm{I}, \mathrm{I}_{\mathrm{C}}, \mathrm{T}, \mathrm{R}\right) \in \mathrm{R}_{+}{ }^{6}\right\}$. The following steps are followed to establish the positive invariance of $\Omega$ (i.e. All solutions in $\Omega$ remain in $\Omega$ for all time). The rate of change of the total population, which is obtained by addingall the equations in the model (1) is given by,

$$
\frac{d N(t)}{d t}=\pi-\mu V(t)-\mu S(t)-\left(\mu+\delta_{1}\right) I(t)-\mu I_{c}(t)-(\mu+\delta) T(t)-\mu R(t)
$$

It is simple to observe that for $N>\frac{\pi}{\mu}, \frac{d N}{d t}<0$ using a standard comparison theorem [7], it is possible to show boundedness as follows.

$$
\begin{aligned}
& \dot{N}(t)=\dot{S}(t)+\dot{V}(t)+\dot{I}(t)+\dot{I}_{C}(t)+\dot{T}(t)+\dot{R}(t) \\
& \dot{N}(t)=\pi+\mu\left(S+V+I+I_{C}+T+R\right)-\delta_{1} I-\delta T \\
& \dot{N}(t)=\pi-\mu N(t)-\delta_{1} I-\delta T \\
& \dot{N}(t) \leq \pi-\mu N(t) \\
& \therefore \dot{N}(t) \leq \pi-\mu N(t)
\end{aligned}
$$

When we solve this first order linear differential equation, we get

$$
\begin{gathered}
N(t) \leq \frac{\pi}{\mu}+e^{-\mu t}\left(N(0)-\frac{\pi}{\mu}\right), \text { since } e^{-\mu t} \leq 1, \text { for } t \geq 0 \\
\text { If } N(0) \leq \frac{\pi}{\mu}, \text { then } N(t) \leq \frac{\pi}{\mu} \text { for } t \geq 0
\end{gathered}
$$

Thus, the model can be considered as being epidemiologically and mathematically well posed [8]. Therefore, every solution of the model (1) with initial conditions in $\Omega$ remains there for $\mathrm{t}>0$.

This result can be summarized as a lemma below.

Lemma 3.1. The region $\Omega$ is positively invariant for the model (1) with non-negative conditions in $R_{+}{ }^{6}$.

Positivity of the Solution
For the typhoid fever Transmission model (1) to be epidemiologically meaningful, it is important to prove that all its state variables are non-negative for all time.

Let $\Omega=\left\{\left(\mathrm{S}, \mathrm{V}, \mathrm{I}, \mathrm{I}_{\mathrm{C}}, \mathrm{T}, \mathrm{R}\right) \in \mathrm{R}_{+}{ }^{6:} \mathrm{S}_{0}>0, \mathrm{~V}_{0}>0, \mathrm{I}_{0}>0, \mathrm{I}_{\mathrm{C} 0}>\right.$ $\left.0, \mathrm{~T}_{0}>0, \mathrm{R}_{0}>0\right\}$ then the solution of $\left\{\left(\mathrm{S}, \mathrm{V}, \mathrm{I}, \mathrm{I}_{\mathrm{C}}, \mathrm{T}, \mathrm{R}\right)\right\}$ are positive for $t \geq 0$. This can be proved as below.

From the system of the differential equation (1) let us take the first equation,

$$
\begin{aligned}
& \frac{d V(t)}{d t}=\varphi S(t)-(\omega+(1-\varepsilon) \lambda) V(t) \\
& \Rightarrow \frac{d V(t)}{d t} \geq-(\omega+(1-\varepsilon) \lambda+\mu) V(t) \\
& \Rightarrow \frac{d V(t)}{V(t)} \geq-(\omega+(1-\varepsilon) \lambda+\mu) d t \\
& \int \frac{d V(t)}{V(t)} \geq-\int(\omega+(1-\varepsilon) \lambda+\mu) d t \\
& V(t) \geq V_{0} e^{-(\omega+(1-\varepsilon) \lambda+\mu) t} \geq 0
\end{aligned}
$$

Similiarly, cinsider the second equation of (1) which is

$$
\begin{aligned}
& \frac{d S(t)}{d t}=\pi+\omega V(t)-(\mu+\lambda+\varphi) S(t) \\
& \Rightarrow \frac{d S(t)}{d t} \geq-(\mu+\lambda+\varphi) S(t) \\
& \Rightarrow \frac{d S(t)}{S(t)} \geq-(\mu+\lambda+\varphi) d t \\
& \int \frac{d S(t)}{S(t)} \geq-\int(\mu+\lambda+\varphi) d t \\
& S(t) \geq S_{0} e^{-(\mu+\lambda+\varphi) t} \geq 0
\end{aligned}
$$

By following the same technique it have been showed that $\mathrm{I}(\mathrm{t}) \geq 0, \mathrm{I}_{\mathrm{C} 0}(\mathrm{t}) \geq 0, \mathrm{~T}(\mathrm{t}) \geq 0 \& \mathrm{R}(\mathrm{t}) \geq 0$ for all values of $\mathrm{t}$.

Disease Free-equilibrium

The model (1) has a disease free equilibrium (DFE), obtained by setting the right hand side of the equations in the model to zero and the force of infection
The Basic Reproduction Number

Following Van Den Driessche [22] the basic reproduction number is obtained as the spectral radius of the matrix $\mathrm{FV}^{-1}$ at the DFE is 0.

Definition 1.Basic reproduction number, basic reproduction ratio or basic reproductive rate is defined as the average number of secondary infections that occur when one infective is introduced into a completely susceptible host population [9].

We can calculate the basic reproduction ratio (number) $R_{0}$, using the Van Den Driesseche and Watmough next generation approach. In order to compute the basic reproduction number, it is important to distinguish new infections from all other class transitions in the population. The infected classes are $\mathrm{I}, \mathrm{I}_{\mathrm{C}}$ and $\mathrm{T}$. We can write system (1) as: $\mathbf{x}=\mathrm{F}(\mathrm{x})-\mathrm{V}(\mathrm{x}), \mathrm{V}=\mathrm{V}^{-}-\mathrm{V}^{+}$, where $\mathbf{x}=\left(\mathrm{S}, \mathrm{V}, \mathrm{I}, \mathrm{I}_{\mathrm{C}}, \mathrm{T}, \mathrm{R}\right) . \mathrm{F}$ is the rate of appearance of new infections in each class, $\mathrm{V}^{+}$is the rate of transfer into each class by all other means, and $\mathrm{V}^{-}$ is the rate transfer out of each class. Using system of differential equations below, the underlined terms are new infections in each class.

$$
\begin{aligned}
& \dot{I}(t)=\rho \lambda S(t)+\underline{(1-\epsilon) \lambda V(t)}+\theta I_{C}(t)-A_{1} I(t) \\
& \dot{I_{C}}(t)=\underline{(1-\rho) \lambda S(t)+\tau T(t)}-A_{2} I_{C}(t) \\
& \dot{T}(t)=\eta I(t)+\sigma I_{C}(t)-A_{3} T(t) \\
& \dot{V}(t)=\varphi S(t)-(\omega+\mu+(1-\epsilon) \lambda) V(t) \\
& \dot{S}(t)=\pi+\omega V(t)-(\mu+\varphi+\lambda) S(t) \\
& \dot{R}(t)=\alpha I(t)+\gamma T(t)-\mu R(t)
\end{aligned}
$$


The associated matrices $\mathrm{F}(\mathrm{x})$ for new infections terms, and $\mathrm{V}(\mathrm{x})$ for the remaining transition terms are respectively given by,

$$
F(x)=\left(\begin{array}{c}
\rho \lambda S+(1-\epsilon) \lambda V \\
(1-\rho) \lambda S \\
0 \\
0 \\
0 \\
0
\end{array}\right)
$$

$$
F=\left(\begin{array}{ccc}
\frac{\rho c \beta S^{D}}{N^{D}}+\frac{(1-\epsilon) c \beta V^{D}}{N^{D}} & \frac{\rho c \beta k_{1} S^{D}}{N^{D}}+\frac{(1-\epsilon) c \beta k_{1} V^{D}}{N^{D}} & \frac{\rho c \beta k_{2} S^{D}}{N^{D}}+\frac{(1-\epsilon) c \beta k_{2} V^{D}}{N^{D}} \\
\frac{(1-\rho) c \beta S^{D}}{N^{D}} & \frac{(1-\rho) c \beta k_{1} S^{D}}{N^{D}} & \frac{(1-\rho) c \beta k_{2} S^{D}}{N^{D}}
\end{array}\right)
$$

$$
=\left(\begin{array}{ccc}
\frac{c \beta(\rho \varphi(\omega+\varphi)+(1-\epsilon) \varphi \mu)}{\varphi \mu+\pi(\omega+\varphi)} & \frac{c \beta k_{1}(\rho \varphi(\omega+\varphi)+(1-\epsilon) \varphi \mu)}{\varphi \mu+\pi(\omega+\varphi)} & \frac{c \beta k_{2}(\rho \varphi(\omega+\varphi)+(1-\epsilon) \varphi \mu)}{\varphi \mu+\pi(\omega+\varphi)} \\
\frac{c \beta \pi(1-\rho)(\omega+\varphi)}{\varphi \mu+\pi(\omega+\varphi)} & \frac{c \beta k_{1} \pi(1-\rho)(\omega+\varphi)}{\varphi \mu+\pi(\omega+\varphi)} & \frac{c \beta k_{2} \pi(1-\rho)(\omega+\varphi)}{\varphi \mu+\pi(\omega+\varphi)} \\
0 & 0 & 0
\end{array}\right)
$$

Similarly, partial differentiation of (5) with respect to $\mathrm{I}, \mathrm{I}_{\mathrm{C}}$ and $\mathrm{T}$ at at $\mathrm{E}_{0}$ gives

$$
V=\left(\begin{array}{llc}
A_{1} & -\theta & 0 \\
0 & -A_{2} & -\tau \\
-\eta & -\sigma & A_{3}
\end{array}\right)
$$

The basic reproduction number of Typhoid fever model is defined, following Van den Driessche and Watmough, as the spectral radius of the next generation matrix, $\mathrm{FV}^{-1}$ and it is given by:

$$
V(x)=\left(\begin{array}{c}
A_{1} I-\theta I_{C} \\
A_{2} I_{C}-\tau T \\
A_{3} T-\eta I-\sigma I_{C} \\
(\omega+\mu+(1-\epsilon) \lambda) V-\varphi S \\
(\mu+\varphi+\lambda) S-\omega V-\pi \\
\mu R-\alpha I-\gamma T
\end{array}\right)
$$

Evaluating the partial derivatives of (4) at $\mathcal{E}_{0}$. and bearing in mind that system (1) has three infected classes, namely I, $\mathrm{I}_{\mathrm{C}}$ and $\mathrm{T}$, we obtain 


$$
\begin{aligned}
& \rightarrow \pi+\omega V^{*}-\left(\mu+\phi+\lambda^{*}\right) S^{*}=0 \\
& \rightarrow S^{*}=\frac{\pi+\omega V^{*}}{\mu+\phi+\lambda^{*}} \\
& \therefore S^{*}=\frac{\pi\left(\omega+\phi+\lambda^{*}\right)}{\left(\mu+\phi+\lambda^{*}\right)\left(\omega+\phi+\lambda^{*}\right)-\phi \omega} \\
& \dot{I}(t)=\rho \lambda S(t)+(1-\epsilon) \lambda V(t)+\theta I_{C}(t)-A_{1} I(t)=0 \\
& \rightarrow \rho \lambda^{*} S^{*}+(1-\epsilon) \lambda^{*} V^{*}+\theta I_{C}^{*}-A_{1} I^{*}=0 \\
& \therefore I^{*}=\frac{1}{A_{1}}\left(\rho \lambda^{*} S^{*}+(1-\epsilon) \lambda^{*} V^{*}+\theta I_{C}^{*}\right) \\
& \dot{I_{C}}(t)=(1-\rho) \lambda S(t)+\tau T(t)-A_{2} I_{C}(t)=0 \\
& \rightarrow(1-\rho) \lambda^{*} S^{*}+\tau T^{*}-A_{2} I_{C}^{*}=0 \\
& \therefore I_{C}^{*}=\frac{1}{A_{2}}\left((1-\rho) \lambda^{*} S^{*}+\tau T\right) \\
& \dot{T}(t)=\eta I(t)+\sigma I_{C}(t)-A_{3} T(t)=0 \\
& \rightarrow \eta I^{*}+\sigma I_{C}^{*}-A_{3} T^{*}=0 \\
& \therefore T^{*}=\frac{1}{A_{3}}\left(\eta I^{*}+\sigma I_{C}^{*}\right) \\
& \dot{R}(t)=\alpha I(t)+\gamma T(t)-\mu R(t)=0 \\
& \rightarrow \alpha I^{*}+\gamma T^{*}-\mu R^{*}=0 \\
& \therefore R^{*}=\frac{1}{\mu}\left(\alpha I^{*}+\gamma T^{*}\right)
\end{aligned}
$$

Where, * indicates the populations of each variable at the equilibrium point. Let the population in each class at the steady state be denoted by $\mathrm{V}^{*}, \mathrm{~S}^{*}, \mathrm{I}^{*}, \mathrm{I}_{\mathrm{C}}{ }^{*}, \mathrm{~T}^{*}$ and $\mathrm{R}^{*}$. Then, the force of infection at the fixed points $\lambda^{*}$ is non-negative roots of $\lambda^{*}=\frac{c \beta}{N^{*}}\left(I^{*}+k_{1} I_{c}^{*}+k_{2} T^{*}\right)$

\section{The Optimal Control Model}

Optimal Control (OC) is the process of determining control and state trajectories for a dynamic system over a period of time in order to minimize a performance index. Basic optimal control (OC) problem in Lagrange form can be written as:-

$$
\max _{U} J(x(t), u(t))=\int_{t_{0}}^{t_{f}} f(t, x(t), u(t)) d t
$$

$$
\text { s.t. } \quad \dot{x}(t)=g(t, x(t), u(t))
$$

$x\left(t_{0}\right)=x_{0}$ where $\mathrm{J}(\mathrm{x}(\mathrm{t}), \mathrm{u}(\mathrm{t}))$ is performance index or cost functional, $\mathrm{x}(\mathrm{t})$ state variable, $\mathrm{u}(\mathrm{t})$ a set of control variable from admissible set $\mathrm{U}$ in time $t$ with $\mathrm{t}_{0} \leq \mathrm{t} \leq \mathrm{t}_{\mathrm{f}} \mathrm{x}\left(\mathrm{t}_{\mathrm{f}}\right)$ could be free, which means that the value of $\mathrm{x}\left(\mathrm{t}_{\mathrm{f}}\right)$ is unrestricted, or could be fixed, i.e, $x\left(t_{f}\right)=x_{f}$.

The possible interventions for Typhoid fever disease can be categorized as prevention with vaccination, sanitation and personal hygiene, through antibiotic medication of infectious individuals as well as screen or separate and treat carriers. In this paper, we are taking these interventions as control measures on the transmission dynamics of Typhoid fever.

1) Typhoid fever can be prevented through proper personal hygiene and sanitation such as regularly washing hands with soap and warm water as well as take care of taking contaminated foods and waters. In addition to this, it is possible to prevent the transmission of the disease through Vaccination. Let the current percentage of vaccinating susceptible individuals be $\phi_{0}$ for some $\varphi_{0}>0$ to protect susceptible from typhoid infection and let also assume that the control function $\mathrm{u}_{1}(\mathrm{t})$ measures the percentage of additional susceptible individuals being vaccinated per unit of time. The cost of vaccinating individuals becomes expensive as the proportion of non-Vaccinated individuals gets smaller. So we can add the term $\left(\frac{V}{S}\right)^{m}$ as a coefficient for $\mathrm{u}_{1}^{2}(\mathrm{t})$.

Where, $\mathrm{V}$ represents vaccinated individuals where as $\mathrm{S}$ represents non-vaccinated population and $\mathrm{m}$ is any positive constant integer. Numerical investigations suggested to take $\mathrm{m}=10$ for the best fit. Therefore, we took $\mathrm{m}=10$. Numerical investigations suggested that unrealistic vaccination scheme when the vaccination term was allowed to have the more standard quadratic form $m=2$ [9]. Then its application in the dynamics is modeled by simply replacing the parameter $\phi$ in (1) by $\phi_{0}+\mathrm{u}_{1}(\mathrm{t})$. But due to limitation of resources $\mathrm{u}_{1}(\mathrm{t})$ is restricted to its maximum, Its maximum vaccination rate $\phi_{\max }>0$ or $\phi_{\max }$ is maximum attainable value of $\mathrm{u}_{1}(\mathrm{t})$ at time $\mathrm{t}$, where $0 \leq \phi_{0}+\mathrm{u}_{1} \leq \phi_{\max } \leq 1[5]$.

2) Typhoid fever can usually be successfully treated with a course of antibiotic medication at hospitals or at home. Any blood, stool or urine samples can help to determine the strain of the infection during diagnosis. A course of antibiotic tablets may be prescribed for 7 to 14 days to take at home and recover from infection. Where as if the patient has severe symptoms of typhoid fever, such as persistent vomiting, sever diarrhea or a swollen stomach, hospital admission usually recommended. In hospitals, infected individuals will have antibiotic injections and they also be given fluids and nutrients directly into a vein through an intravenous drip. Most of the patients respond well to hospital treatment and 
improve within three to five days. However, it may be several weeks until patients become well enough to leave hospital. While people who are treated for typhoid fever experience a relapse, which is when disease symptoms return. At this time, further treatment with antibiotic is usually recommended. After symptoms have passed, an individual should have another stool test to check if there are still salmonella Typhi bacteria present in faeces. If there are, that individual becomes a carrier of the typhoid infection and that may need to have a further 28 day course of antibiotics to flush out the bacteria. Hence, by considering this, let assume that the control function $\mathrm{u}_{2}(\mathrm{t})$ measures the rate at which, additional infectious individuals recruited to treated class at any time $t$. If the current percentage of treating infectious individuals is $\eta_{0}$, then this control will be seen in the dynamics as $\eta_{0}+\mathrm{u}_{2}(\mathrm{t})$ instead of $\eta$ in (1). But due to economical and logistic reasons, there are limitations on the maximum rate at which individuals are recruited to get treatment at each time period. Thus, $\eta_{\text {max }}$ represent the maximum rate of recruitment for treatment of infected individuals.

Hence, $0 \leq \eta_{0}+\mathrm{u}_{2}(\mathrm{t}) \leq \eta_{\max } \leq 1$.

Even if it is difficult and challenging to screen or identify carriers from susceptible populations, it is possible to bring behavior change on infectious individuals to check whether the bacteria totally removed from their faeces or not at the end of treatment period. Assume that the current percentage of screening or identifying carriers and bring them to treatment is $\sigma_{0}$, then this control can be seen in the dynamics as $\sigma_{0}+\mathrm{u}_{3}(\mathrm{t})$ instead of $\sigma$ in (1). Since there are economic and logistic reasons, there are limitations on the maximum rate at which carrier individuals are identified and recruited to get additional treatment at each time period. Thus $\sigma_{\max }$ represent the maximum rate of recruitment for treatment of carriers. Hence, $0 \leq \sigma_{0}+\mathrm{u}_{3}(\mathrm{t}) \leq \sigma_{\max } \leq 1$.

Using the above described control parameters, the system of the disease dynamics can be written as:

$$
\begin{gathered}
\dot{V}(t)=\left(\phi_{0}+u_{1}(t)\right) S(t)-(\omega+(1-\epsilon) \lambda+\mu) V(t) \\
\dot{S}(t)=\pi+\omega V(t)-\left(\phi_{0}+u_{1}(t)\right) S(t)-(\mu+\lambda) S(t) \\
\dot{I}(t)=\rho \lambda S(t)-(1-\epsilon) \lambda V(t) \theta I_{C}(t)-\left(\eta_{0}+u_{2}(t)\right) I(t)-a_{1} I(t) \\
\dot{I_{C}}(t)=(1-\rho) \lambda S(t)+\tau T(t)-\left(\sigma_{0}+u_{3}(t)\right) I_{C}(t)-a_{2} T(t) \\
\dot{T}(t)=\left(\eta_{0}+u_{2}(t)\right) I(t)+\left(\sigma_{0}+u_{3}(t)\right) I_{C}(t)-a_{3} T(t) \\
\dot{R}(t)=\alpha I(t)+\gamma T(t)-\mu R(t)
\end{gathered}
$$

Thus, with (9) and given initial population size of each compartment $\mathrm{V}_{0}, \mathrm{~S}_{0}, \mathrm{I}_{0}, \mathrm{I}_{\mathrm{C} 0}, \mathrm{~T}_{0}, \mathrm{R}_{0}$; Our main goal is to find or propose the best strategy in terms of either in combination or independent efforts of Vaccination, treatment Infective, screening or identification as well as treatment of carriers that will minimize the total number of new infections in the planning period and minimize those people who will die from the infectious and treatment classes while at the same time minimize the cost of Vaccination, treatment of Infective and screening as well as treatment of carrier population. We made the optimal control problem a fixed terminal time problem because most governments cannot continue the implementation of the interventions indefinitely; rather they want the disease eradicated or driven below specified level within a set time frame. If we know the initial populations size $\left(\mathrm{V}_{0}, \mathrm{~S}_{0}, \mathrm{I}_{0}, \mathrm{I}_{\mathrm{C} 0}, \mathrm{~T}_{0}, \mathrm{R}_{0}\right)$ and the control trajectory, i.e., the values of $\mathrm{u}(\mathrm{t})$ over the whole time interval $0<\mathrm{t}<\mathrm{T}$, then we can integrate (9) to get the state trajectory, i.e., the values of $\mathrm{V}(\mathrm{t}), \mathrm{S}(\mathrm{t}), \mathrm{I}(\mathrm{t}), \mathrm{I}_{\mathrm{C}}(\mathrm{t}), \mathrm{T}(\mathrm{t})$ and $\mathrm{R}(\mathrm{t})$ over the same time interval. We want to choose the control trajectory so that the state and control trajectories minimize the objective functional, or simply the objective function [12].

$$
J\left(u_{1}, u_{2}, u_{3}, u_{4}\right)=\int_{0}^{t_{f}}\left(C_{1} I(t)+C_{2} I_{c}(t)+\frac{B_{1}}{2}\left(\frac{V}{S}\right)^{m} u_{1}^{2}(t)+\frac{B_{2}}{2} u_{2}^{2}(t)+\frac{B_{2}}{2} u_{3}^{2}(t)\right) d t
$$

where the constants $\mathrm{C}_{1}, \mathrm{C}_{2}$ and $\mathrm{B}_{\mathrm{i}}, \mathrm{i}=1,2,3$ can be considered as values that will balance the units of measurement and also may indicate the importance of one type of intervention over the other. $\mathrm{C} 1 \mathrm{I}$ and C2IC represent the number of infectious and the number of carriers respectively, whereas $\frac{B_{1}}{2}\left(\frac{V}{S}\right)^{m} u_{1}^{2}, \frac{B_{2}}{2} u_{2}^{2}, \frac{B_{3}}{2} u_{3}^{2}$ represents the costs of the Vaccine, treatment cost for infectious individuals, treatment cost of carriers and the cost related to screening or identification of carriers respectively. The Vaccination cost could include the cost of the Vaccine, the Vaccine storage cost, costs of lab tests that could used to determine a person's immune status prior to Vaccination, other related overheads, etc. The treatment cost could include the cost of the medical tests and diagnosis, drug cost, hospitalization cost, etc. The cost function variables are squared to amplify the effects of 
large variations and to de-emphasize contributions of small variations. Since implementation of any public health intervention has increased costs with reaching higher fraction of the population, we take a non-linear cost function like the quadratic. So we seek to find an optimal controls $u_{1}^{*}, u_{2}^{*}, u_{3}^{*}$ such that

$$
J\left(u_{1}^{*}, u_{2}^{*}, u_{3}^{*}\right)=\min _{U} J\left(u_{1}, u_{2}, u_{3}\right)
$$

Where $U=\left\{\left(u_{1}(t), u_{2}(t), u_{3}(t)\right) \in R^{3} \mid u_{1}(t), u_{2}(t), u_{3}(t)\right.$ Are Lebesgue integrable and (11)

$$
\left.\mathrm{u}_{1}(\mathrm{t}) \in\left(0, \phi_{\max }-\phi_{0}\right), \mathrm{u}_{2}(\mathrm{t}) \in\left(0, \eta_{\max }-\eta_{0}\right), \mathrm{u}_{3}(\mathrm{t}) \in\left(0, \sigma_{\max }-\sigma_{0}\right)\right\}
$$

Existence and Characterization of Optimal Control Solution

Theorem 4.1. (Existence of optimal control solution). There exists an optimal control $u_{1}{ }^{*}(t), u_{2}{ }^{*}(t), u_{3}{ }^{*}(t)$ and corresponding solutions $V^{*}, S^{*}, I^{*}, I_{C}{ }^{*}, T^{*}$ and $R^{*}$ and to the state initial value problem (9) - (11) that minimizes $J\left(u_{1}, u_{2}\right.$, $u_{3}$ ) over $U$.

Proof. The non-trivial requirements on the set of admissible controls $U$ and on the set of end conditions are verified from Fleming \& Rishel's theorem.

A. The set of all solutions to system (9) - (11) with corresponding control functions in $\mathrm{U}$ is non-empty.

B. The state system can be written as a linear function of the control variables with coefficients dependent on time and the state variables.

C. The integrand $\mathrm{L}$ in (10) from objective function with

$$
L=C_{1} I(t)+C_{2} I_{c}(t)+\frac{B_{1}}{2}\left(\frac{V}{S}\right)^{m} u_{1}^{2}(t)+\frac{B_{2}}{2} u_{2}^{2}(t)+\frac{B_{3}}{2} u_{3}^{2}(t)
$$

is convex on $\mathrm{U}$ and additionally satisfies $\mathrm{L}(\mathrm{x}, \mathrm{u}, \mathrm{t}) \geq \delta_{1}$ $\left|\left(u_{1}, u_{2}, u_{3}\right)\right|^{\beta}-\delta_{2}$ where $\delta_{1}>0$ and $\beta>0$.In order to establish condition A, we refer to Picard-Lindelof's theorem from [23]. If the solutions to the state equations are bounded and if the state equations are continuous and Lipschitz in the state variables, then there is a unique solution corresponding to every admissible control $\mathrm{U}$.

It is indicated that the total population is bounded below by a positive number $\mathrm{N}_{0}$ and bounded above by $\frac{\pi}{-}$ as well as each of the state variables are bounded. With the bounds established above, it follows that the state system is continuous and bounded. It is equally direct to show the boundedness of the partial derivatives with respect to the state variables in the state system, which establishes that the system is Lipschitz with respect to the state variables [24]. This completes the proof that condition A holds. Condition B is verified by observing the linear dependence of the state equations on controls $\mathrm{u}_{1} ; \mathrm{u}_{2}$ and $\mathrm{u}_{3}$. Finally to verify condition $\mathrm{C}$, since any combinations of convex functions are also convex the integrand $\mathrm{u}_{1}{ }^{2}(\mathrm{t}), \mathrm{u}_{2}{ }^{2}(\mathrm{t}), \mathrm{u}_{2}{ }^{3}(\mathrm{t})$ are convex on U. Since a linear combination of convex functions is also convex the integrand $\mathrm{L}(\mathrm{x}, \mathrm{u}, \mathrm{t})$ is convex on $\mathrm{U}$.

To prove the bound on the $\mathrm{L}$ we note that by the definition of $U$, we have

$$
\begin{gathered}
B_{3} u_{3}^{2} \leq B_{3} \sin c e u_{3} \in[0,1], \frac{B_{3}}{2} u_{3}^{2} \leq \frac{B_{3}}{2}, \Rightarrow \frac{B_{3}}{2} u_{3}^{2}-\frac{B_{3}}{2} \leq 0 \\
L(x, u, t)=C_{1} I(t)+C_{2} I_{C}(t)+\frac{B_{1}}{2} u_{1}^{2}(t)\left(\frac{V}{S}\right)^{m}+\frac{B_{2}}{2} u_{2}^{2}(t)+\frac{B_{3}}{2} u_{3}^{2} \\
\geq \frac{B_{1}}{2} u_{1}^{2}(t)\left(\frac{V}{S}\right)^{m}+\frac{B_{2}}{2} u_{2}^{2}(t)+\frac{B_{3}}{2} u_{3}^{2}(t)-\frac{B_{3}}{2} \\
\Rightarrow L(x, u, t) \geq \min \left(\frac{B_{1}}{2}\left(\frac{V}{S}\right)^{m}, \frac{B_{2}}{2}, \frac{B_{3}}{2}\right)\left(u_{3}^{2}+u_{2}^{2}+u_{3}^{2}\right)-\frac{B_{3}}{2} \\
\Rightarrow L(x, u, t) \geq \min \left(\frac{B_{1}}{2}\left(\frac{V}{S}\right)^{m}, \frac{B_{2}}{2}, \frac{B_{3}}{2}\right)\left|\left(u_{3}^{2}, u_{2}^{2}, u_{3}^{2}\right)\right|^{2}-\frac{B_{3}}{2} \\
\therefore L(x, u, t) \geq \delta_{1}\left|\left(u_{1}, u_{2}, u_{3}\right)\right|^{\beta}-\delta_{2}
\end{gathered}
$$

Where

$$
\delta_{1}=\min \left(\frac{B_{1}}{2}\left(\frac{V}{S}\right)^{m}, \frac{B_{2}}{2}, \frac{B_{3}}{2}\right), \delta_{2}=\frac{B_{3}}{2} \& \beta=2 .
$$

The necessary conditions that an optimal solution must satisfy come from Pontryagin's maximum principle (PMP). This principle converts (9)-(11) into a problem of minimizing a Hamiltonian; $\mathrm{H}$ with respect to $\mathrm{u}_{1}, \mathrm{u}_{2}, \mathrm{u}_{3}$. Then the Hamiltonian is given by

$$
\begin{aligned}
& H(x, u, h . t)= C_{1} I(t)+C_{2} I_{C}(t)+\frac{B_{1}}{2}\left(\frac{V}{S}\right)^{m} u_{1}^{2}(t)+\frac{B_{2}}{2} u_{2}^{2}(t)+\frac{B_{3}}{2} u_{3}^{2}(t)+\sum_{i=1}^{6} h_{i} f_{i} \\
& \Rightarrow H(x, u, h . t)=\left\{C_{1} I(t)+C_{2} I_{C}(t)+\frac{B_{1}}{2} u_{1}^{2}(t)\left(\frac{V}{S}\right)^{m}+\frac{B_{2}}{2} u_{2}^{2}(t)+\frac{B_{3}}{2} u_{3}^{2}(t)\right. \\
&+h_{1}\left[\left(\phi_{0}+u_{1}(t)\right) S(t)-(\omega+(1-\epsilon) \lambda+\mu) V(t)\right] \\
&+h_{2}\left[\pi+\omega V(t)-\left(\phi_{0}+u_{1}(t)\right) S(t)-(\mu+\lambda) S(t)\right]
\end{aligned}
$$




$$
\begin{gathered}
+h_{3}\left[\rho \lambda S(t)+(1-\epsilon) \lambda V(t)+\theta I_{C}(t)-\left(\eta_{0}+u_{2}(t)\right) I(t)-a_{1} I(t)\right] \\
+h_{4}\left[(1-\rho) \lambda S(t)+\tau T(t)-\left(\sigma_{0}+u_{3}(t)\right) I_{C}(t)-a_{2} I_{C}(t)\right] \\
+h_{5}\left\lfloor\left(\eta_{0}+u_{2}(t)\right) I(t)+\left(\sigma+u_{3}(t)\right) I_{C}(t)-a_{3} T(t)\right] \\
\left.+h_{6}[\alpha I(t)+\gamma T(t)-\mu R(t)]\right\}
\end{gathered}
$$

When we substitute the values of $\lambda=\frac{c \beta}{N(t)}\left(I(t)+k_{1} I_{C}(t)+k_{2} T(t)\right)$, then we can write the Hamiltonian as:

$$
\begin{gathered}
\Rightarrow H(x, u, h . t)=\left\{C_{1} I(t)+C_{2} I_{C}(t)+\frac{B_{1}}{2} u_{1}^{2}(t)\left(\frac{V}{S}\right)^{m}+\frac{B_{2}}{2} u_{2}^{2}(t)+\frac{B_{3}}{2} u_{3}^{2}(t)\right. \\
+h_{1}\left[\left(\phi_{0}+u_{1}(t)\right) S(t)-\left(\omega+(1-\epsilon) \frac{c \beta}{N(t)}\left(I(t) k_{1} I_{C}(t)\right)+\mu\right) V(t)\right] \\
+h_{2}\left[\pi+\omega V(t)-\left(\phi_{0}+u_{1}(t)\right) S(t)-\left(\mu+\frac{c \beta}{N(t)}\left(I(t)+k_{1} I_{C}(t)+k_{2} T(t)\right)\right) S(t)\right] \\
+h_{3}\left[\begin{array}{c}
\left.\rho \frac{c \beta}{N(t)}\left(I(r)+k_{1} I_{C}(t) k_{2} T(t)\right) S(t)+(1-\epsilon) \frac{c \beta}{N(t)}\left(I(t)+k_{1} I_{C}(t)+k_{2} T(t)\right) V(t)\right] \\
+\theta I_{C}(t)-\left(\eta_{0}+u_{1}(t)\right) I(t)-a_{1} I(t)
\end{array}\right. \\
+h_{4}\left[\begin{array}{c}
\left.(1-\rho) \frac{c \beta}{N(t)}\left(I(t)+k_{1} I_{C}(t)\right) S(t)+\tau T(t)-\left(\sigma_{0}+u_{3}(t)\right) I_{C}(t)-a_{2} I_{C}(t)\right] \\
+h_{5}\left[\left(\eta_{0}+u_{2}(t)\right) I(t)+\left(\sigma+u_{3}(t)\right) I_{C}(t)-a_{3} T(t)\right] \\
\left.+h_{6}[\alpha I(t)+\gamma T(t)-\mu R(t)]\right\}
\end{array}\right.
\end{gathered}
$$

Where, $f_{i}$ is the right hand side of the differential equation of $\mathrm{i}^{\text {th }}$ state variable of $(9) \mathrm{x}=\left(\mathrm{S}, \mathrm{V}, \mathrm{I}, \mathrm{I}_{\mathrm{C}}, \mathrm{T}, \mathrm{R}\right), \mathrm{u}=\left(\mathrm{u}_{1}, \mathrm{u}_{2}, \mathrm{u}_{3}\right), \mathrm{h}=\left(\mathrm{h}_{1}\right.$, $\left.\mathrm{h}_{2}, \mathrm{~h}_{3}, \mathrm{~h}_{4}, \mathrm{~h}_{5}, \mathrm{~h}_{6}\right)$. If $\left(\mathrm{u}_{1}{ }^{*}, \mathrm{u}_{2}{ }^{*}, \mathrm{u}_{3}{ }^{*}\right)$ is an optimal control yet to be determined, then from Pontryagins Maximum Principe we have: The minimum conditions:

$$
\begin{gathered}
\frac{\partial H}{\alpha u_{i}}=0, i=1,2,3, \\
\bullet \frac{\partial H}{\alpha u_{1}}=0 \Rightarrow B_{1}\left(\frac{V}{S}\right)^{m} u_{1}(t)+h_{1} S-h_{2} S=0 \\
\therefore u_{1}(t)=\frac{1}{B_{1}}\left(\frac{V}{S}\right)^{-m}\left(h_{2}-h_{1}\right) \\
\bullet \frac{\partial H}{\alpha u_{2}}=0 \Rightarrow B_{2} u_{2}(t)-h_{3} I+h_{5} I=0 \\
\therefore u_{2}(t)=\frac{1}{B_{2}}\left(h_{3}-h_{5}\right) I \\
\bullet \frac{\partial H}{\alpha u_{3}}=0 \Rightarrow B_{3} u_{3}(t)-h_{4} I_{C}+h_{5} I_{C}=0 \\
\therefore u_{3}(t)=\frac{1}{B_{3}}\left(h_{4}-h_{5}\right) I_{C}
\end{gathered}
$$


The adjoint equation:

i. $\quad \dot{h}_{1}=-\frac{\partial H}{\partial S}$

$$
\begin{gathered}
=\frac{c \beta\left(I+k_{1} I_{C}+k_{2} T\right)\left(V+I+I_{C}+T+R\right)\left(h_{2}-\rho h_{3}-(1-\rho) h_{4}\right)}{\left(S+V+I+I_{C}+T+R\right)^{2}} \\
+m \frac{B_{1}}{2} u_{1}^{2}(t) \frac{V^{m}}{S^{m+1}}+\left(\varphi_{0}+u_{1}(t)\right)\left(h_{2}-h_{1}\right)+\frac{c \beta(1-\epsilon)\left(h_{3}-h_{1}\right)\left(I+k_{1} I_{C}+k_{2} T\right) V}{\left(S+V+I+I_{C}+T+R\right)^{2}}
\end{gathered}
$$

ii. $\dot{h}_{2}=-\frac{\partial H}{\partial V}$

$$
\begin{gathered}
=\frac{c \beta(1-\epsilon)\left(h_{1}-h_{3}\right)\left(I+k_{1} I_{C}+k_{2} T\right)}{S+V+I+I_{C}+T+R}+\frac{c \beta\left(I+k_{1} I_{C}+k_{2} T\right)\left(\rho h_{3}+(1-\rho) h_{4}\right) S}{\left(S+V+I+I_{C}+T+R\right)^{2}} \\
+h_{1}(\omega+\mu)+\frac{c \beta(1-\epsilon)\left(h_{3}-h_{1} V\right)\left(I+k_{1} I_{C}+k_{2} T\right)}{\left(S+V+I+I_{C}+T+R\right)^{2}}-m \frac{B_{1}}{2} u_{1}^{2}(t) \frac{V^{m-1}}{S^{m}}
\end{gathered}
$$

iii. $h_{3}=-\frac{\partial H}{\partial I}$

$$
\begin{gathered}
=-C_{1}+\frac{c \beta(1-\epsilon)\left(h_{1}+h_{3}\right)\left(S+V+\left(1-k_{1}\right)+I_{C}+\left(1-k_{2}\right) T+R\right) V}{\left(S+V+I+I_{C}+T+R\right)^{2}}+a_{1} h_{3} \\
+\left(\eta_{0}+u_{2}(t)\right)\left(h_{3}-h_{5}\right)+\frac{c \beta\left(h_{2}-\rho h_{3}+(1-\rho) h_{4}\right)\left(S+V+\left(1-k_{1}\right) I+\left(1-k_{2}\right) T+R\right) S}{\left(S+V+I+I_{C}+T+R\right)^{2}}
\end{gathered}
$$

iv. $\dot{h}_{4}=-\frac{\partial H}{\partial I_{C}}$

$$
\begin{gathered}
=-C_{2}+\frac{c \beta(1-\epsilon)\left(h_{1}+h_{3}\right)\left(k_{1} S+k_{1} V+\left(k_{1}-1\right) I+\left(k_{1}-k_{2}\right) T+k_{1} R\right) V}{\left(S+V+I+I_{C}+T+R\right)^{2}} \\
+\frac{c \beta\left(h_{2}-\rho h_{3}+(1-\rho) h_{4}\right)\left(k_{1} S+k_{1} V+\left(k_{1}-1\right) I+\left(k_{1}-k_{2}\right) T+k_{1} R\right) V}{\left(S+V+I+I_{C}+T+R\right)^{2}} \\
+\left(\sigma_{0}+u_{3}(t)\right)\left(h_{4}-h_{5}\right)+a_{2} h_{4}-\theta h_{3}
\end{gathered}
$$

v. $\dot{h}_{5}=-\frac{\partial H}{\partial T}$

$$
\begin{gathered}
=\frac{c \beta\left(h_{2}-\rho h_{3}+(1-\rho) h_{4}\right)\left(k_{2} S+k_{2} V+\left(k_{2}-1\right) I+\left(k_{2}-k_{1}\right) I_{C}+k_{2} R\right) S}{\left(S+V+I+I_{C}+T+R\right)^{2}}+a_{3} h_{5} \\
+\frac{c \beta(1-\epsilon)\left(h_{1}+h_{3}\right)\left(k_{2} S+k_{2} V+\left(k_{2}-1\right) I+\left(k_{2}-k_{1}\right) I_{C}+k_{2} R\right) V}{\left(S+V+I+I_{C}+T+R\right)^{2}}-\gamma h_{6}
\end{gathered}
$$

vi. $\dot{h}_{6}=-\frac{\partial H}{\partial R}$

$$
\begin{gathered}
=\frac{c \beta\left(\rho h_{3}+(1-\rho) h_{4}-h_{2}\right)\left(I+k_{1} I_{C}+k_{2} T\right) S}{\left(S+V+I+I_{C}+T+R\right)^{2}}+\mu h_{6} \\
+\frac{c \beta(1-\epsilon)\left(h_{3}-h_{1}\right)\left(I+k_{1} I_{C}+k_{2} T\right) V}{\left(S+V+I+I_{C}+T+R\right)^{2}}-\gamma h_{6}
\end{gathered}
$$




\section{Numerical Simulation and Results}

We need to show/simulate the result by using fourth order Runge-Kutta method. The process begins with an initial guess on the control variable. Then, the state equations are simultaneously solved forward in time and the adjoint equations are solved backward in time. The control is updated by inserting the new values of states and adjoints into its characterization, and the process is repeated until convergence occurs. Considering $\mathbf{x}$ and $\lambda$ vector approximations for the state and the adjoint respectively. The main idea of the algorithm is described as follows:

Step 1:- Make an initial guess for $u$ over the interval $u=0$ is almost always sufficient) and store the initial guess as $u$;

Step 2:- Using the initial condition $\mathrm{x}\left(\mathrm{t}_{0}\right)=\mathrm{x}_{0}$ and the values for $\mathrm{u}$ stored in step 1 , solve $\mathrm{x}$ forward in time according to its differential equation in the optimality system;

Step 3:- Using the transversality condition $\lambda\left(t_{f}\right)=0$ and the values for $\mathrm{u}$ and $\mathrm{x}$, solve $\mathrm{x}$ backward in time according to its differential equation in the optimality system;

Step 4:- Update $\mathrm{u}$ by entering the new $\mathrm{x}$ values into the characterization of the optimal control;

Step5:-Verify convergence: if the variables are sufficiently close to the corresponding in the previous iteration, then output the current values as solutions, else return to Step 2 .

To examine the impact or role of each control strategy on eradication of the disease, we used the following strategies.

Applying Vaccination only $\left(\mathrm{u}_{1}\right)$ as an intervention,

Applying the treatment of infectious only $\left(\mathrm{u}_{2}\right)$ as an intervention,

Applying screening and treatment of carriers only $\left(\mathrm{u}_{3}\right)$ as an intervention,

Implementing Vaccination $\left(\mathrm{u}_{1}\right)$ and treatment of infections $\left(\mathrm{u}_{2}\right)$ intervention,

Implementing Vaccination $\left(\mathrm{u}_{1}\right)$ and screening as well as treatment of carriers $\left(\mathrm{u}_{3}\right)$ intervention,

Implementing treatment of infectious $\left(\mathrm{u}_{2}\right)$ and that of carriers after screening $\left(\mathrm{u}_{3}\right)$ intervention.

Implementation of all controls

Implementation without a control

For simulation purpose the author used the following initial value as well as coefficients of the state and controls. $\mathrm{S}_{0}=1000, \mathrm{I}_{0}=200, \mathrm{~V}_{0}=50, \mathrm{I}_{\mathrm{C} 0}=100, \mathrm{~T}_{0}=60, \mathrm{R}_{0}=70$.

Table 1. Parameter values for the model.

\begin{tabular}{llll}
\hline Parameters & Description & Values & Source \\
\hline$\pi$ & Recruitment rate & 0.0077 & {$[20]$} \\
$\beta$ & Probability of disease transmission & 0.12 & Assumed \\
$\mu$ & Natural death rate & 0.028 & Assumed \\
$\varepsilon$ & Efficacy of the vaccine & 0.80 & {$[11]$} \\
$\phi$ & Vaccination rate & 0.90 & Assumed \\
$\omega$ & Waning rate & 0.33 & Assumed \\
$\rho$ & Proportion of susceptible becomes & 0.072 & {$[20]$} \\
$\mathrm{C}$ & newly infected & 10 & {$[26]$} \\
$\mathrm{K}_{1}, \mathrm{k}_{2}$ & Contacts rate & $1-1.2$ & Varied \\
$\delta_{1}$ & Modification parameter & 0.07 & Assumed \\
\hline
\end{tabular}

\begin{tabular}{llll}
\hline Parameters & Description & Values & Source \\
\hline \multirow{\gamma}{*}{$\eta$} & infectious & & \\
$\eta$ & Disease induced deaths of carriers & 0.05 & Assumed \\
$\sigma$ & Treatment rate of invectives & 0.09 & Assumed \\
$\gamma$ & Treatment rate of carriers & 0.015 & Assumed \\
$\alpha$ & Recovery rate of treated & 0.025 & Assumed \\
$\theta$ & individuals & 0.015 & Assumed \\
\hline
\end{tabular}

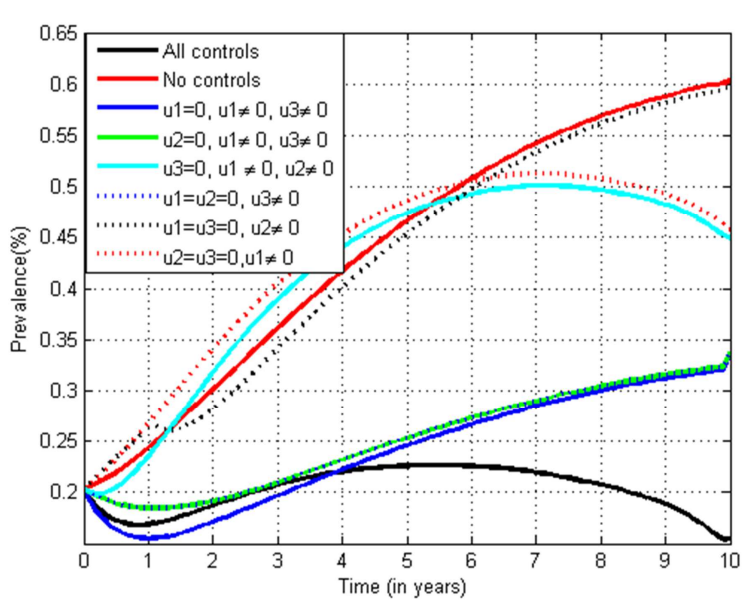

Figure 1. The graph of prevalence.

The above figure 1 indicates that, the prevalence of the disease decrease and will be zero due to the implementation of all control measures (implementation of vaccination, treatment of infection, screening and treatment of carriers) with minimum cost as indicated in figure 2 below. On the other way, the absence of any control intervention rises up the prevalence as indicated in figure 1 above.

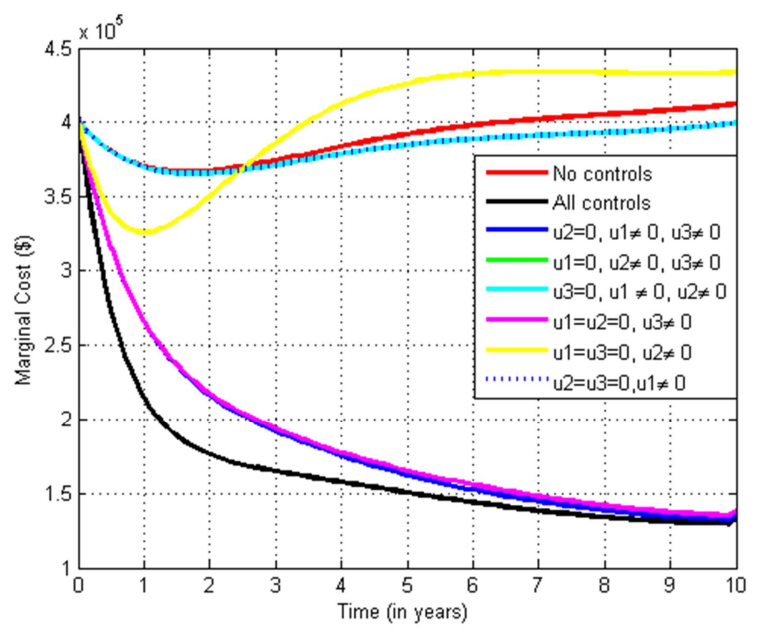

Figure 2. Marginal cost of intervention for all control measure.

Since we need an interventions that decrease the prevalence and the number of infective with minimum cost of interventions, the above figure indicates that the implementation of all controls helps to decrease the number of infective and the prevalence of the disease with the least or 
minimum cost of interventions.

The number of carrier population decreased significantly due to implementation of the following control measures. These include implementation of all controls, implementation of screening of carriers and treatment of both infectious and carriers in the absence of vaccination; implementation of vaccination and screening as well as treatment of carriers in the absence of treatment for infection; and implementation of screening and treatment of carriers in the absence of the other interventions.

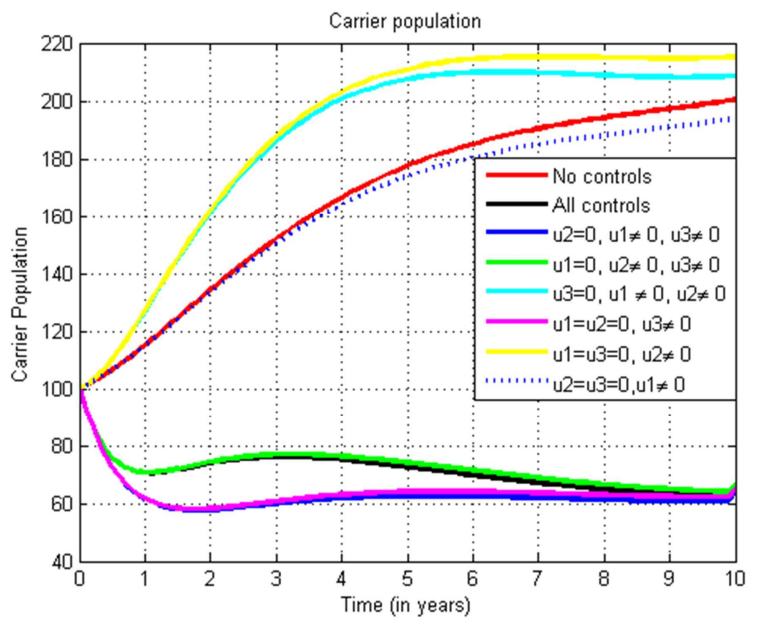

Figure 3. Graph of carrier population

In the other corner, implementation of only treatment of infections; implementation of vaccination and treatment of infective; only implementation of vaccination; and absence of any control will not decrease the number of carrier population. Since we need the interventions or control measures that can decrease the number of carrier population to the optimal level at the same time decrease the cost of interventions, the best control strategy to decrease the number of carriers as well as the cost of interventions indicated in the figure 3 above an implementation of vaccination, treatment of infection, screening and treatment of carriers.

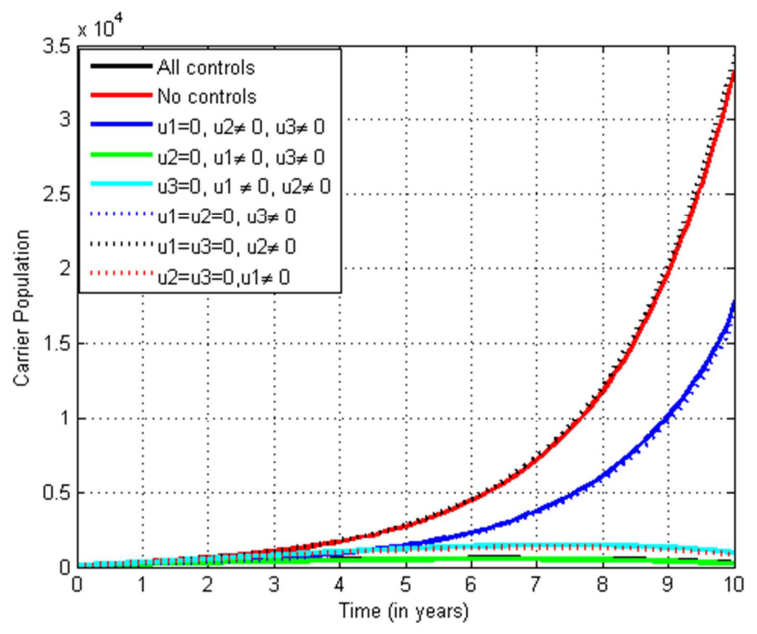

Figure 4. the graph of Typhoid infectious population.
The number of infectious individuals falls down and approaches zero as indicated in the above figure 4 due to the presence of the following control measures. These are the presence of all controls; implementation of vaccination and screening as well as treatment of carriers. But an interventions that could decrease the prevalence of the disease with minimum cost of interventions as indicated in figure 1 and figure 2 above is an implementation of all controls. Therefore, based on the simulation result of the model, the author concludes and recommends the following.

\section{Conclusions}

In this study, a deterministic mathematical model for the transmission dynamics of Typhoid fever disease is proposed. The qualitative analysis of the model shows that the solution of the model is bounded and positive and also the equilibrium points of the model are obtained. The basic reproduction number is calculated for the model analytically. Formulation of optimal controls and the existence of the optimal control solution was analyzed. Three control measures such as vaccination; treatment of typhoid infectious population, screening and treatment of carriers were applied to the model to investigate the roles of control measures independently and as combination. The proposed control strategies investigated numerically and results are displayed numerically using fourth order Runge-Kutta method from Matlab software. Implementation of Vaccination only as preventive strategy, implementation of treatment for infective as well as identify and treat carrier populations independently will not help to control the transmission of Typhoid fever. Individuals should have to be convinced properly to visit health centers soon after the end of treatment, whether their faeces is free from the bacteria or not for further treatment. Simulation result indicated that implementation of the combined control measures helps to decrease the prevalence of the disease. Therefore, the author concludes that adequate implementation of all control measures (Vaccination, treatment of infections, screen and treat those infected individuals) would be a very cost effective mechanism to decrease the prevalence or eradicate the disease from the community. This paper is hypothetical and requires detailed study involving sensitivity analysis and parameter estimations to improve model predictions.

\section{Acknowledgements}

The Author thank our colleagues from Kotebe Metropolitan University who provided insight and expertise that greatly assisted this research.

\section{References}

[1] Roumagnac P. (2006), Evolutionary history of Salmonella typhi, Science, 314, 1301-304.

[2] WHO (2011), Guidelines for the Management of Typhoid Fever.

[3] WHO (2007), Background paper on vaccination against typhoid fever using New Generation Vaccines presented at the SAGE. 
[4] Anwar E, Goldberg E, Fraser A, Acosta CJ, Paul M. and Leibovici L. (2014), Vaccination for preventing typhoid fever. The Cochrane Database of Systematic Reviews.

[5] Gaff H. and Schaefer E. (2009), Optimal control applied to vaccination and treatment Strategies for various epidemiological models, mathematical bio-sciences and engineering, Volume 6, Number 3, 469492.

[6] Hethcote H. (2000), The mathematics of infectious diseases. SIAM Review. 42 (4): 599-53.

[7] Kassa, S. M., Ouhinou, A. (2014), The impact of selfprotective measures in the optimal Interventions for controlling infectious diseases of human population. J. Math. Biol.

[8] Lakshmikantham V., Leela S, and Martynyuk A. (1989), Stability Analysis of NonlinearSystems. Marcel Dekker, Inc., New York and Basel.

[9] Ma S. and Xia Y. (2009), Mathematical understanding of infectious disease dynamics, World Scientific Publishing Co. Pte. Ltd. vol. 16.

[10] Awoke, T. D.; Kassa, S. M. Optimal Control Strategy for TBHIV/AIDS Co-Infection Model in the Presence of Behaviour Modification. Processes 2018, 6, 48.

[11] Aweke T. D. and Kassa S. M. (2015), Impacts of vaccination and behavior change in the optimal intervention strategy folr controlling the transmission of Tuberculosis, Springer International Publishing Switzerland 2015, CIM Series in Mathematical Sciences, Vol. 2, pp. 15-32.

[12] Sethi S. P. and Thompson G. L. (2000), Optimal control theory: Applications to management science and economics, Kluwer, Boston, second edition.

[13] Tunde Tajudeen Yusuf and Francis Benyah (2012), Optimal control of vaccination and treatment for an SIR epidemiological model, World Journal of Modelling and Simulation, Vol. 8 No. 3, pp. 194-204.

[14] Cook JH. (2010), Are Cholera and typhoid vaccines a good investment for slums in Kolkata, India. Pediatr. Infect. Dis. J; 9 (5): 485-496.

[15] Mushayabasa S. (2011), Impacts of Vaccines on controlling Typhoid Fever in Kassena Nankana district of upper east region of Ghana: Insight from a mathematical model,
Journalof modern mathematics and statistics, 5 (2): 54-59.

[16] Pitzer VE, Bowles CC, Baker S, Kang G, Balaji V, Farrar J. (2014), is predicting the Impactof vaccination on the transmission dynamics of typhoid in South Asia: A Mathematicalmodeling study. PLoS Negl Dis, 8 (1): 1-12.

[17] Kalajdzievska D. and Li M. (2011), Modeling the effects of carriers on transmission dynamics of infectious disease, Mathematical Bioscience and Engineering, Num. 3, Vol. 8, 711-722.

[18] Mushayabasa S.(2014)., Modeling the impact of optimal screening on typhoid dynamics, Int. J. Dynam. Control, Springer-Verlag Berlin Heidelberg.

[19] Kariuki S. (2008), Typhoid fever in sub-saharan Africa; Challenges of diagnosis and management of infections. Journal of infection in Developing Countries. 2 (6): 443-447.

[20] Kgosimore M. and G. R. Kelatlhegile G. (2016), Mathematical analysis of Typhoid Infectionwith treatment, Journal of Mathematical Science: Advances and applications, Vol. 40, 75-91.

[21] Rachel B. Slayton, Kashmira A. Date3 and Eric D. Mintz (2013), Vaccination for typhoid fever in Sub-Saharan Africa, Human Vaccines \& Immunotherapeutics 9: 4, 903-906.

[22] Van den Driessche, P. and Watmough J. (2002), Reproduction numbers and sub-thresholdendemic equilibria for compartmental models of disease transmission. Mathematical Bio-sciences, Volume 180, pp: 29-48.

[23] Waaler H., Gese A., and Anderson S. (1962), The use of mathematical models in the study of the epidemiology of tuberculosis, Am. J. Publ. Health, 52, pp. 1002-1013.

[24] Coddington, E. A. (1961), An Introduction to Ordinary Differential Equations. Prentice-Hall, Englewood Cliffs.

[25] Cvjetanovic B., Grab B. and Uemura K. (1971), Epidemiological model of typhoid fever and its use in the planning and evaluation of antityphoid immunization and sanitation Programs, Bull. Org. Mond. Sante (45), 53-75.

[26] Ghosh M., Chandra P., Sinha P. and Shukla J. B (2004), Modelling the spread of Carrierdependent infectious diseases with an environmental effect, Appl. Math. Comp. 